\title{
Coronagraph design for an extreme adaptive optics system with spatially filtered wavefront sensing on segmented telescopes
}

\begin{abstract}
Anand Sivaramakrishnan, Russell B. Makidon, Remi Soummer, Bruce A. Macintosh, Mitchell Troy, et al.
\end{abstract}

Anand Sivaramakrishnan, Russell B. Makidon, Remi Soummer, Bruce A. Macintosh, Mitchell Troy, Gary A. Chanan, James P. Lloyd, Marshall D. Perrin, James R. Graham, Lisa Poyneer, Andrew I. Sheinis, "Coronagraph design for an extreme adaptive optics system with spatially filtered wavefront sensing on segmented telescopes," Proc. SPIE 5490, Advancements in Adaptive Optics, (25 October 2004); doi: 10.1117/12.551939

Event: SPIE Astronomical Telescopes + Instrumentation, 2004, Glasgow, United Kingdom 


\title{
Coronagraph design for an Extreme Adaptive Optics System with Spatially-Filtered Wavefront Sensing on Segmented Telescopes
}

\author{
Anand Sivaramakrishnan $^{a, g}$, Russell B. Makidon ${ }^{a, g}$, Rémi Soummer ${ }^{a, g}$, Bruce A. Macintosh ${ }^{b, g}$, \\ Mitchell Troy ${ }^{c, g}$, Gary A. Chanan ${ }^{d, g}$, James P. Lloyd ${ }^{e, g}$, Marshall D. Perrin ${ }^{f, g}$, James R. \\ Graham $^{f, g}$, Lisa Poyneer ${ }^{b, g}$, and Andrew Sheinis ${ }^{g}$ \\ ${ }^{a}$ Space Telescope Science Institute, 3700 San Martin Drive, Baltimore, MD, USA \\ ${ }^{b}$ Lawrence Livermore National Laboratory 7000 East Ave Livermore, CA 94551, USA \\ ${ }^{c}$ Jet Propulsion Laboratory, Pasadena, CA 91109, USA \\ ${ }^{d}$ Department of Physics and Astronomy, University of California, Irvine CA 92697, USA \\ ${ }^{e}$ Astronomy Department, California Institute of Technology, Pasadena CA 91125, USA

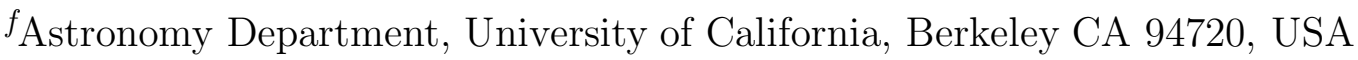 \\ ${ }^{g}$ Center for Adaptive Optics, University of California, 1156 High Street, Santa Cruz CA 95064
}

\begin{abstract}
High dynamic range coronagraphy targeted at discovering planets around nearby stars is often associated with monolithic, unobstructed aperture space telescopes. With the advent of extreme adaptive optics (ExAO) systems with thousands of sensing and correcting channels, the benefits of placing a near-infrared coronagraph on a large segmented mirror telescope become scientifically interesting. This is because increased aperture size produces a tremendous gain in achievable contrast at the same angular distance from a point source at Strehl ratios in excess of $90 \%$ (and at lower Strehl ratios on future giant telescopes such as the Thirty Meter Telescope). We outline some of the design issues facing such a coronagraph, and model a band-limited coronagraph on an aperture with a Keck-like pupil. We examine the purely diffractive challenges facing the eXtreme AO Planetary Imager (XAOPI) given the Keck pupil geometry, notably its inter-segment gap spacing of $6 \mathrm{~mm}$. Classical Lyot coronagraphs, with hard-edged occulting stops, are not efficient enough at suppressing diffracted light, given XAOPI's scientific goal of imaging a young Jupiter at a separation as close as $0.15 \operatorname{arcseconds}(4 \lambda / D$ at $H$ on Keck) from its parent star. With a 4000 channel ExAO system using an anti-aliased spatially-filtered wavefront sensor planned for XAOPI, we wish to keep diffracted light due to coronagraphic design at least as low as the noise floor set by AO system limitations. We study the band-limited Lyot coronagraph (BLC) as a baseline design instead of the classical design because of its efficient light suppression, as well as its analytical simplicity. We also develop ways of investigating tolerancing coronagraphic mask fabrication by utilizing the BLC design's mathematical tractability.
\end{abstract}

Keywords: segmented mirror coronagraphy, Lyot coronagraphy, band-limited coronagraph, Extreme AO, Keck, TMT

\section{INTRODUCTION}

Ground-based imaging of faint companions and structure around nearby bright stars faces two severe problems. First, one must use a high-order adaptive optics system good enough to correct for atmospheric phase (and possibly amplitude) aberrations to produce a superb diffraction-limited image, and then one has to suppress the central bright star's light in order to discern underlying faint structure.

Further author information: (Send correspondence to A.S.: E-mail: anand@stsci.edu ) 
The benefits of using a large telescope for this problem are detailed by Lloyd. ${ }^{1}$ Hence one should look to use the largest existing telescopes to image planetary companions and disks around nearby stars that are bright enough to provide the requisite signal for excellent measurement of the stellar wavefront. The largest telescopes today are the $10 \mathrm{~m}$ diameter Keck telescopes, which are segmented aperture telescopes. Future giant telescopes are also segmented designs (e.g., Nelson ${ }^{2}{ }^{2}$ Dierickx $\left.{ }^{3}\right)$. The existence of these segments, with their inter-segment gaps and complicated point-spread functions (PSFs) (Chanan and Troy, ${ }^{4}$ Yaitskova and Dohlen ${ }^{5}$ ), as well as individual segment figure errors, complicates solutions of the second problem mentioned above: the problem of suppressing the diffracted light of the central star.

We examine this problem here: given the Keck aperture geometry, with no phase or transmission errors in the optics or atmosphere, we explore the effects of the inter-segment gaps on a coronagraph that would suppress all the central object light were the aperture continuous. While secondary support obstructions are relevant to the coronagraphic design problem, we focus here on the ramifications of segmentation. Our goal is to reduce the coronagraphic PSF to below $10^{-7}$ of the peak value of an unocculted star passing through the same Lyot stop. The spatially filtered wavefront sensor ExAO system planned for the eXtreme Adaptive Optics Planetary Imager (XAOPI) (Poyneer and Macintosh ${ }^{6}$ ) is expected to produce sufficient image quality to make this level of coronagraphic mage suppression desirable.

\section{BASIC CORONAGRAPHIC THEORY}

A mathematical description of the fundamentals of Lyot coronagraphy (Lyot, ${ }^{7}$ Lyot $^{8}$ ) can be found in Sivaramakrishnan et al., ${ }^{9}$ Aime et al., ${ }^{10}$ Aime et al., ${ }^{11}$ and Soummer et al., ${ }^{12}$ and references therein. We summarize the notation used here briefly.

\subsection{Aperture illumination function}

The telescope entrance aperture and all phase effects in a monochromatic wave front impinging upon the optical system are described by a real aperture illumination function $A(x, y)$ multiplied by a unit modulus function $A_{\phi}(x, y)=e^{i \phi(x, y)}$. Aperture plane coordinates are $(x, y)$ in units of the wavelength of light, and image plane coordinates are $(\xi, \eta)$ in radians. Deviations from a plane wave are described by a real wave front phase function $\phi(x, y)$ (in radians).

We assume that the electric field in the image plane is described by the Fourier transform (FT) of the field in the aperture plane $\left(e . g .,{ }^{13}\right)$. We write the FT of a function $A$ as $a$, and the FT of $\phi$ as $\Phi$, by changing case to indicate a transform. The aperture illumination function with phase aberrations is

$$
A_{\text {aber }}=A A_{\phi},
$$

with a corresponding 'amplitude-spread function' (ASF) of $a_{a b e r}=a * a_{\phi}$ (where $*$ denotes the convolution operation, and $a_{\phi}$ is the FT of $\left.A_{\phi}(x, y)\right)$. The ASF is proportional to the electric field in the image plane, and is a complex-valued function of angular image plane coordinates $(\xi, \eta)$.

\subsection{The Lyot plane field}

A coronagraphic stop is modelled by multiplying the image field by a stop function $w(\xi, \eta)$, so the electric field strength just after the image plane stop is $a_{a b e r} w$. When describing a Lyot coronagraph it is sometimes helpful to introduce a 'mask function' $m$ by the definition

$$
w(\xi, \eta) \equiv 1-m(\xi, \eta) .
$$

When $m$ is unity at the origin the mask is opaque at its center. We can write the electric field at the Lyot plane as $E_{\text {Lyot }}=A(x, y) *(\delta(x, y)-M(x, y))$, or

$$
E_{\text {Lyot }}(x, y)=A(x, y)-A(x, y) * M(x, y) .
$$


Classical Lyot
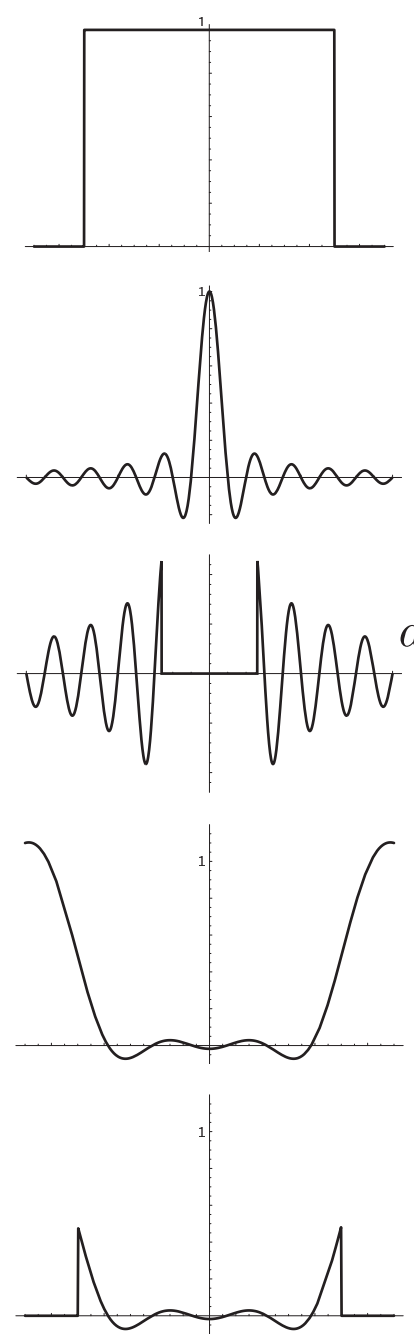

Band Limited Lyot

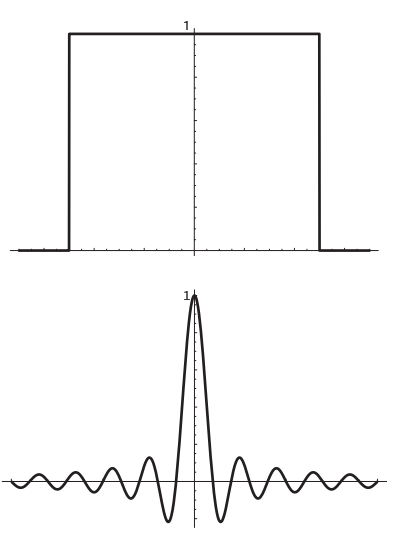

$A(x, y)$

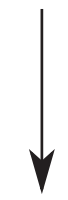

$$
a(\eta, \xi)
$$
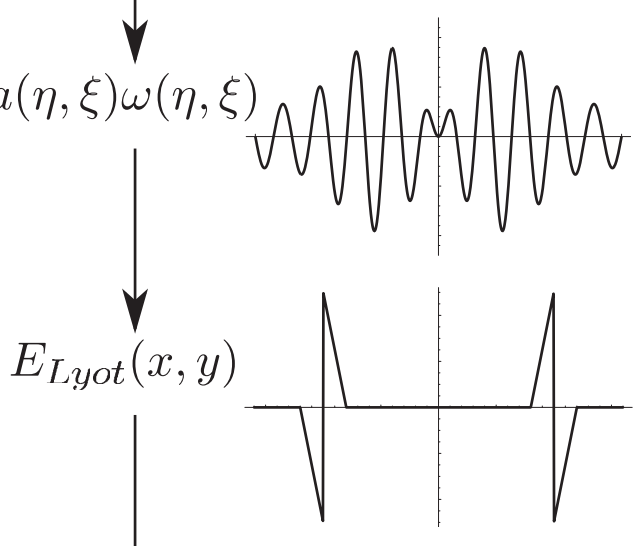

$$
E_{\text {Lyot }}^{\text {stop }}(x, y)
$$

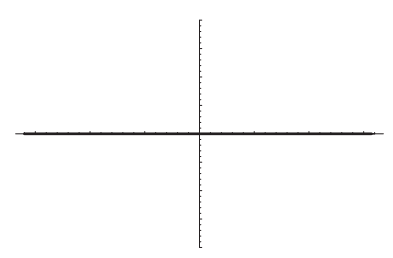

Figure 1. A sketch of the classical Lyot coronagraph (left), and the 1-sinc "top-hat" band-limited coronagraph operating on a perfectly flat incoming wavefront. The top row shows the same aperture function used in both examples, viz., a clear unobscured entrance aperture. The second row shows the field strength at the image plane. The third row shows the field strength immediately after passage through the focal plane occulting stop. The classical Lyot, on the left, has a hard-edged, opaque occulter. The band-limited coronagraph (on the right) has an occulter that is opaque only at its center. The fourth row shows the field strength in the Lyot pupil plane before a Lyot stop is applied, and the final row shows the field after the Lyot stop in the Lyot pupil plane. The band-limited coronagraph blocks all the incoming on-axis light if the wavefront is unaberrated (bottom right). The classical Lyot design with a finite image plane occulting stop (bottom left) never blocks all incoming light unless the Lyot stop is completely opaque. 


\subsection{Band-limited Lyot coronagraphs}

In some sense coronagraphs only suppress the perfect part of an incoming on-axis wavefront. Because of this, they are not usually useful for imaging within a few diffraction widths of the central star when adaptive optics systems deliver Strehl ratios lower than about $80-85 \%$ (see Sivaramakrishnan et al. ${ }^{9}$ for details). At very large separations, ten or more diffraction widths away from the central PSF, they can provide substantial benefits even at very low Strehl ratios (e.g., Smith and Terrile $\left.{ }^{14}\right)$

A classical Lyot coronagraph, with its hard-edged aperture and opaque, hard-edged, focal plane occulter, does suppress enough light to be useful for diffraction-limited stellar coronagraphy when the image Strehl ratio is between $80-90 \%$. They do continue to be useful for PSF suppression at higher Strehl ratios, but when the Strehl ratio starts exceeding $90 \%$, the classical Lyot leaks enough of the perfectly corrected part of the wave into the final image to limit the dynamic range of the coronagraph.

Improved Lyot coronagraph designs which eliminate all, or nearly all, of the on-axis incoming light from the final image exist. These coronagraphs differ from the classical design by smoothing the sharp edges of the stops, either in the image plane, as in the band-limited Lyot coronagraph (Kuchner and Traub ${ }^{15}$ ), or in the entrance pupil, as in the apodized pupil Lyot coronagraph (Soummer, Aime and Falloon ${ }^{12}$ ).

Kuchner and Traub ${ }^{15}$ used an image plane mask function $m$ which is band-limited. This means that there is a minimum positive value of $b$ such that the mask function's Fourier Transform, $M$, satisfies the property $M(x, y)=0$ if $\left|x^{2}+y^{2}\right|>b^{2}$. In the terminology of Fourier theory, the width of the bandpass is $b$ (even though $b$ is actually a physical distance in pupil space). We select the mask function in its transform (pupil) space, $(x, y)$, rather than in physical (image) space, $(\xi, \eta)$, even though it is applied in the image plane in any real coronagraph. If the telescope diameter is $D$, then the characteristic scale of the mask function is $D / b$ resolution elements in the image plane. Each resolution element is $\lambda / D$ in angular extent, where $\lambda$ is the wavelength of the monochromatic light being considered. Although we have already defined $\lambda$ to be unity by our choice of units of length in the pupil plane, we write it explicitly here for clarity. Thus the image plane occulter will be about $D / b$ Airy rings in size.

We define the interior of the aperture as that part of the aperture (or image of the aperture, such as the Lyot plane) which is further than $b$ from any point on the aperture boundary (where $b$ is the bandwidth of the occulter). This is the area of the Lyot pupil which would not be obstructed by a matched Lyot stop in a perfect band-limited coronagraph A Lyot stop which only transmits in the interior of the Lyot plane will reject all on-axis light that was allowed to pass by the focal plane occulter. In the Lyot plane interior the condition

$$
A(x, y)-A(x, y) * M(x, y)=0
$$

holds exactly. The transmission function of the matched Lyot stop is written $A^{\prime}(x, y)$. With this Lyot stop, the coronagraph suppresses all incoming unaberrated on-axis light.

\section{SIMULATIONS}

\subsection{Numerical sampling}

Our simulations utilize simple Fraunhofer propagators between pupil and image planes, which we implemented as fast Fourier transforms (FFTs). The range in interesting scales made for large numerical arrays. With a $10 \mathrm{~m}$ diameter primary mirror, two samples across a $10 \mathrm{~mm}$ inter-segment gap requires 2000 samples. With our available computer memory we were limited to running $4096 \times 4096$ FFT arrays. While Nyquist-sampling of the image plane is not in general fine enough for coronagraphic design studies, we were forced to accept Nyquistsampling in our simulated images in order to study the Keck coronagraphic design problem. We selected 2048 samples across the pupil (which was embedded in a $4096 \times 4096$ zero-padded array), and studied a sequence of increasingly narrow gap sizes, starting from $48 \mathrm{~mm}$ inter-segment gaps — which are we sampled well given our pupil sampling scale of $4.883 \mathrm{~mm}$ per sample - down to $12 \mathrm{~mm}$ gaps (which are poorly sampled) in order to understand the trend as we approached the actual optical inter-segment gap size of $6 \mathrm{~mm}$. The physical gap size is smaller $(4 \mathrm{~mm})$ : the effects of segment edge roll-off (i.e., the difference between the optical and physical gap sizes) was not considered here. 


\subsection{Optical modelling}

We generated direct and coronagraphic imaging PSFs using four configurations: with our aperture sampling scale of $4.883 \mathrm{~mm}$ per sample, the gap sizes we modelled, $12 \mathrm{~mm}, 24 \mathrm{~mm}$, and $48 \mathrm{~mm}$, have 2.46, 4.92, 9.83 samples perpendicularly across a gap (when the gap is aligned with the sampling grid).

Given that the narrowest segment gap modelled is barely sampled, with approximately two samples across the gap, we used the approach and code of Troy and Chanan ${ }^{16}$ to generate the aperture illumination function for the segmented aperture. Since we wish to understand the actual Keck pupil geometry from the coronagraphic standpoint, we chose to look at how the on-axis coronagraphic PSF behaves with decreasing gap size.

Chanan and Troy's representation produces the correct image plane field strength by utilizing an analytical calculation of the expected image field strength of a single segment, combining the Fourier shift theorem and the analytical Fourier transform of a hexagonal segment. This exact field is sampled on a uniform grid and reverse-propagated to the pupil plane with an FFT, with no sampling-induced aliasing.

Starting with this pupil illumination function as the origin of our optical train places only 'in-band' structure into our first numerically generated image. There is no ringing or aliasing in this image due to sampling effects in the first pupil. Unfortunately our subsequent modelling cannot benefit from this ingenious analytical sleight of hand - we simply sampled our image plane occulter on the appropriate angular scale, and multiplied the first image field by the numerical array representing the occulter.

The resultant occulted image was then propagated to the Lyot pupil plane with a forward FFT. There we applied various Lyot stops to the image, to determine how much of the input pupil needs to be blocked by the Lyot stop. We modelled Lyot stops that were 'clear' (undersized on the outer edge of the pupil, and oversized at the inner edge), as well as 'reticulated' Lyot stops with the same inner and outer diameters as the clear Lyot stops, but in addition masked out the segment gaps themselves.

Table 1. Band-Limited Coronagraphic PSF strength $(9 \lambda / D$ mask $)$

\begin{tabular}{rcccc}
\hline \hline Gap size & $0 \mathrm{~mm}$ & $12 \mathrm{~mm}$ & $24 \mathrm{~mm}$ & $48 \mathrm{~mm}$ \\
$0 \lambda / D$ & $6.6 \times 10^{-11}$ & $3.1 \times 10^{-4}$ & $1.0 \times 10^{-3}$ & $3.4 \times 10^{-3}$ \\
$5 \lambda / D$ & $5.0 \times 10^{-12}$ & $5.0 \times 10^{-6}$ & $1.7 \times 10^{-5}$ & $7.1 \times 10^{-5}$ \\
$10 \lambda / D$ & $7.1 \times 10^{-12}$ & $1.8 \times 10^{-7}$ & $5.4 \times 10^{-7}$ & $2.0 \times 10^{-6}$ \\
$15 \lambda / D$ & $2.8 \times 10^{-12}$ & $2.6 \times 10^{-7}$ & $7.4 \times 10^{-8}$ & $2.5 \times 10^{-7}$ \\
$20 \lambda / D$ & $3.4 \times 10^{-12}$ & $1.1 \times 10^{-7}$ & $4.7 \times 10^{-8}$ & $1.6 \times 10^{-7}$ \\
$25 \lambda / D$ & $9.7 \times 10^{-13}$ & $8.0 \times 10^{-9}$ & $7.1 \times 10^{-8}$ & $3.0 \times 10^{-7}$ \\
\hline
\end{tabular}

Table 2. Band-Limited Coronagraphic PSF strength $(12 \lambda / D$ mask $)$

\begin{tabular}{rcccc}
\hline \hline Gap size & $0 \mathrm{~mm}$ & $12 \mathrm{~mm}$ & $24 \mathrm{~mm}$ & $48 \mathrm{~mm}$ \\
$0 \lambda / D$ & $1.3 \times 10^{-10}$ & $3.2 \times 10^{-4}$ & $1.0 \times 10^{-3}$ & $3.4 \times 10^{-3}$ \\
$5 \lambda / D$ & $6.5 \times 10^{-12}$ & $2.5 \times 10^{-7}$ & $7.2 \times 10^{-7}$ & $4.1 \times 10^{-6}$ \\
$10 \lambda / D$ & $2.2 \times 10^{-11}$ & $2.7 \times 10^{-7}$ & $1.0 \times 10^{-6}$ & $2.6 \times 10^{-6}$ \\
$15 \lambda / D$ & $6.3 \times 10^{-13}$ & $4.0 \times 10^{-9}$ & $2.6 \times 10^{-7}$ & $2.2 \times 10^{-6}$ \\
$20 \lambda / D$ & $1.8 \times 10^{-12}$ & $1.0 \times 10^{-8}$ & $1.7 \times 10^{-9}$ & $1.9 \times 10^{-7}$ \\
$25 \lambda / D$ & $4.1 \times 10^{-12}$ & $2.3 \times 10^{-9}$ & $4.1 \times 10^{-8}$ & $2.5 \times 10^{-7}$ \\
\hline
\end{tabular}

Figure 2 shows the various planes of interest in a band-limited coronagraph with a $12 \lambda / D$-sized occulter and matched Lyot stop. The structure of the intensity in the Lyot plane of the optical train with well-sampled gaps is easily understood from the explanations in Sivaramakrishnan et al. ${ }^{9}$ The limitations of our numerical modelling 

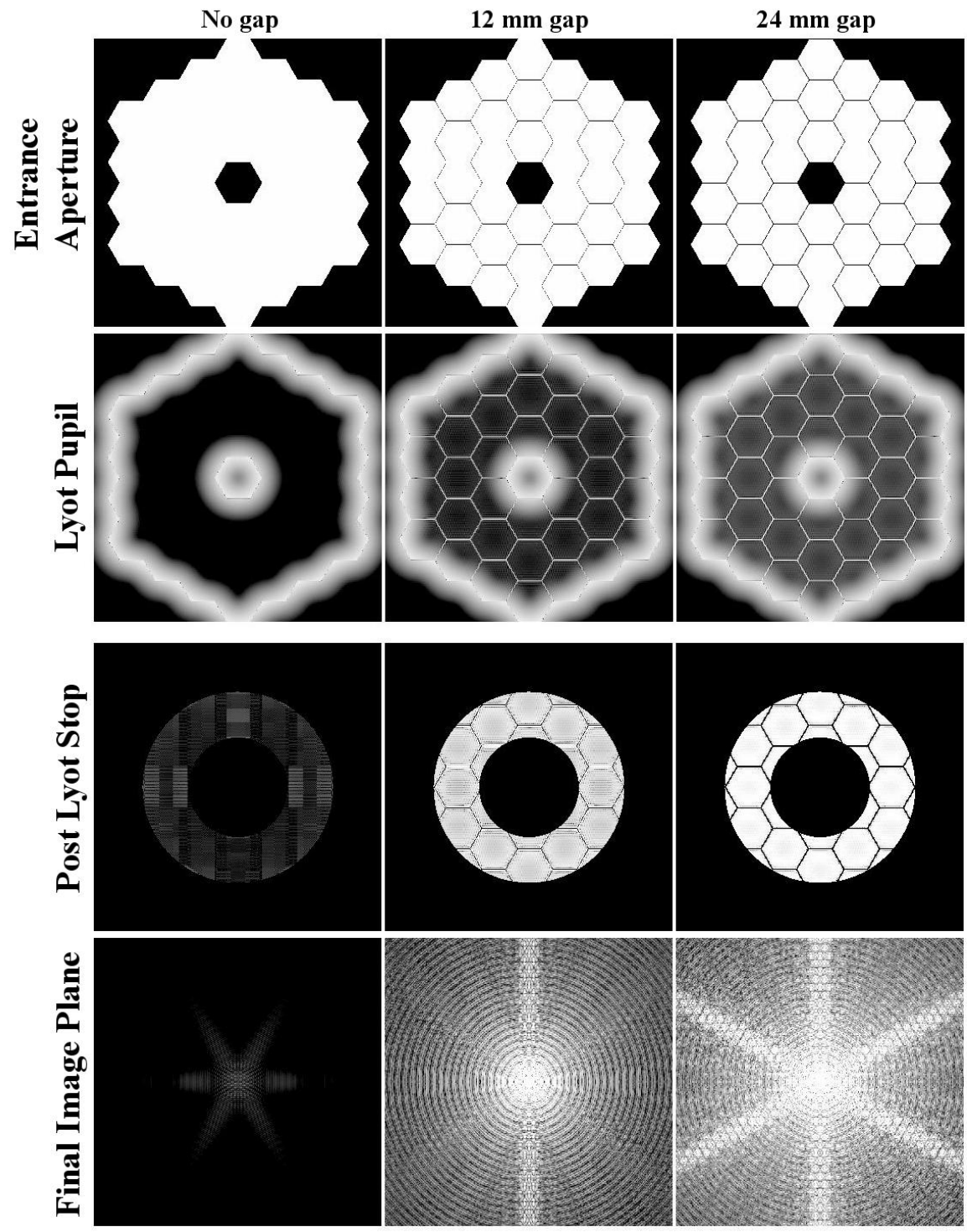

Figure 2. The effect of inter-segment gap size on band-limited Lyot coronagraphs. All three columns show the entrance aperture intensity (top row), the Lyot pupil intensity after the on-axis image is occulted by a band-limited coronagraph with a focal plane occulter transmission profile of $1-J_{1}^{2}(D \theta / 12 \lambda)$ where $\theta$ is angular distance from the PSF core and $J_{1}$ is a Bessel function of the first kind (second row), the stopped down Lyot pupil intensity with the segment gaps masked out with a reticulate structure matching the gap widths (third row), and the final coronagraphic PSF (bottom row, on a logarithmic scale). Each image in a row shares the same display stretch. The PSF is calculated with a sampling pitch of $\lambda / 2 D$. The leftmost column shows the limiting case without inter-segment gaps, the middle column the $12 \mathrm{~mm}$ gap case, and the rightmost the $24 \mathrm{~mm}$ gap case. Images in the second row show that the bright inter-segment gaps in the Lyot plane contain a significant amount of power. Blocking the bright segment gaps in the Lyot pupil improves the final coronagraphic PSF. Also noteworthy is the way the intensity of 'background' light inside the segments increases roughly as the square of the inter-segment gap size. A $6 \mathrm{~mm}$ segment gap configuration must deliver an on-axis PSF intensity less that $10^{-7}$ of an unocculted PSF's peak value after passage through the Lyot stop for a Keck ExAO coronagraph to be able to detect young Jupiters within a few diffraction widths of their parent star. 

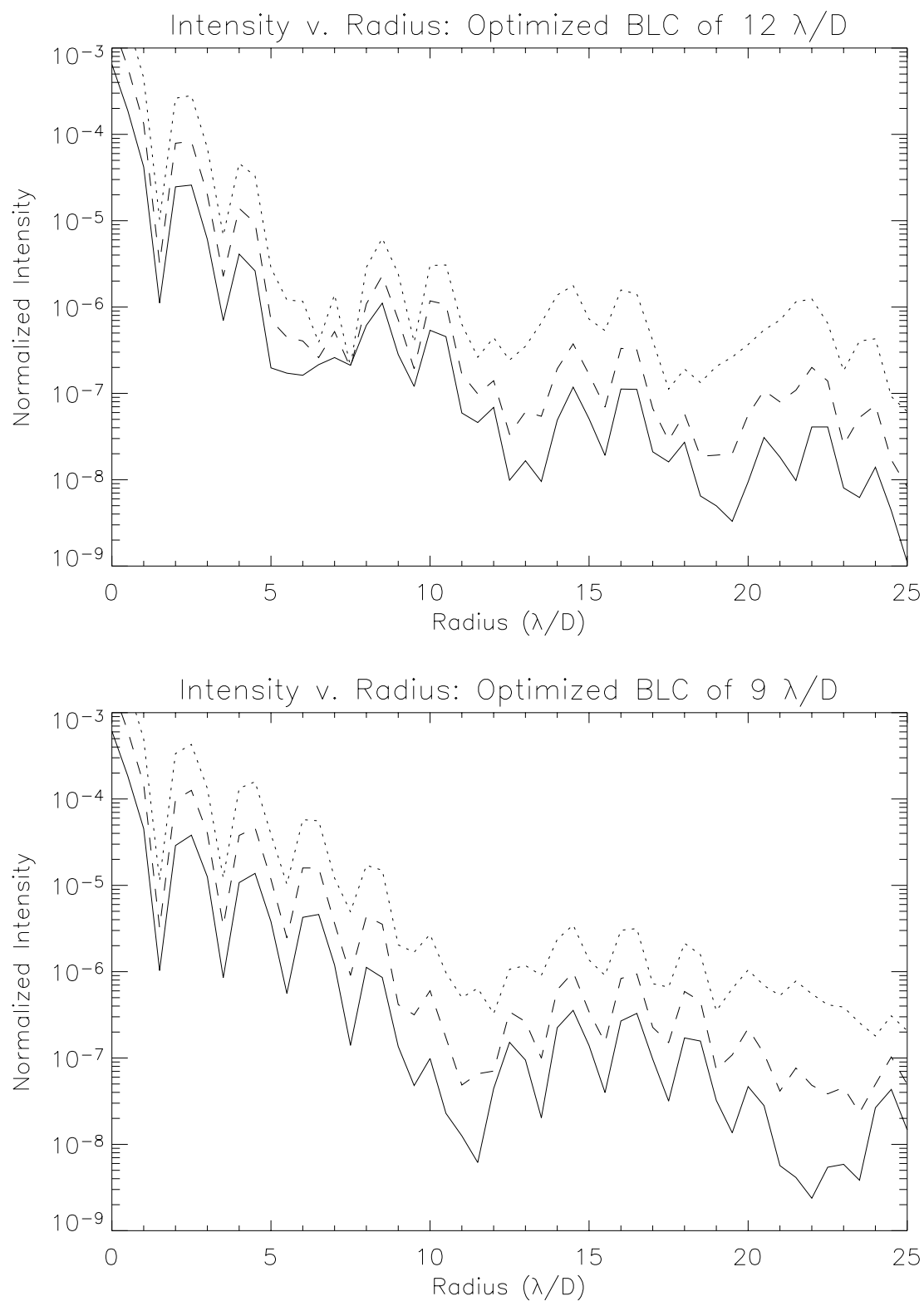

Figure 3. Radial profiles of Keck-like coronagraphic PSFs from band-limited coronagraphs with two different focal plane occulters, without atmospheric or figure errors. The highest of the three curves in each panel (dotted line) corresponds to a $48 \mathrm{~mm}$ inter-segment gap Keck aperture, the middle line is the PSF profile for a $24 \mathrm{~mm}$ gap, the solid line a $12 \mathrm{~mm}$ gap. A Keck coronagraph will have to contend with a $6 \mathrm{~mm}$ gap between Keck segments. The wider occulter (top) may approach the requisite image suppression of $10^{-7}$ (relative to an unocculted PSF with the same Lyot stop) at separations of 4-5 $\lambda / D$ from the central star. The narrower occulter may only go down to $10^{-6}$ of the unocculted but Lyot-stopped PSF. Integration times of these configurations for the same companion detection levels $(e . g ., 5-\sigma)$ will need to be estimated: the throughput of the wider occulter for a point source at a $5 \lambda / D$ separation is less than that of the narrower occulter. Real telescope, instrument, and atmospheric temporal stability may play a role in evaluating which coronagraph will perform better after PSF calibration and subtraction. 
can be seen in the post-Lyot stop image for the no gap case, where the Lyot field starts to pick up structure that is purely numerical artifacts. Quantitative examination of the radial profiles in Figure 3 is somewhat reassuring in that the progression of PSF suppression from the widest inter-segment gap down to the smallest is smooth, although our $\lambda / 2 D$ image plane sampling is still not fine enough to trust completely. Examination of the values in the tabulation of some of the coronagraphic PSF intensities suggests a quadratic dependence of coronagraphic PSF intensity on inter-segment gap width in the cases we studied (see tables 1 and 2).

\section{MASK TOLERANCING}

Kuchner and Traub ${ }^{15}$ presented some analysis of mask fabrication tolerancing. Here we develop a simple formalism that emphasises image plane structure rather than using their interferometric parallel.

The perfect mask function $m(\xi, \eta)$ may be realized in hardware with some error, $\epsilon(\xi, \eta)$, so equation (2) becomes

$$
w(\xi, \eta)=1-m(\xi, \eta)-\epsilon(\xi, \eta) .
$$

The Lyot plane field is then written as

$$
E_{\text {Lyot }}(x, y)=A(x, y)-A(x, y) * M(x, y)-A(x, y) * E(x, y),
$$

where $A$ is the aperture illumination function without phase aberrations, as used in equation (1). For the band-limited coronagraph this reduces to

$$
E_{\text {Lyot }}(x, y)=-A^{\prime}(x, y)(A(x, y) * E(x, y))
$$

in the Lyot plane interior, after the Lyot stop. Here $E(x)$ is the Fourier transform of the error in mask manufacturing, $\epsilon$. The final ASF of the coronagraph, which should be identically zero in the absence of errors (according to the simple theory), is

$$
a_{l e a k}(\xi, \eta)=-a^{\prime} *(a \epsilon) .
$$

While opacity errors are described by a real function $\epsilon(\xi, \eta)$, phase errors in the mask may also occur.

If $\epsilon(\xi, \eta)$ contains errors in transmission that vary slowly with position in the image plane (compared to the resolution of the optical system, $\lambda / D)$, then over the scale of the first image, there will be a transmission error $\epsilon_{0} \equiv \epsilon(0,0)$. This is just a leak: equation (8) shows that the residual light leaking through this coronagraph is $\epsilon_{0}^{2} a^{\prime} a^{\prime *}$ (we use the fact that $a * a^{\prime}=a^{\prime}$ here, which is true for unapodized apertures and Lyot stops).

Mask throughput errors on a scale much smaller than the telescope resolution (or the resolution of the system with a Lyot stop in place, to be precise) will be washed out by the convolution of $\epsilon$ with $a^{\prime}$, as long as they average to zero over a scale length smaller than $\lambda / D$. Thus the most important errors in mask transmission are those on the scale of the Airy pattern itself.

In the monochromatic case, mask transmission errors are only relevant where there is significant light in the image plane, i.e., the brighter portions of the Airy pattern. With broad-band imaging the first, noncoronagraphic, image will typically be devoid of dark areas near its core. These conclusions agree with common sense, but a theoretical framework for tolerancing band-limited masks is a useful tool to develop.

\section{DISCUSSION}

We demonstrate that a band-limited Lyot coronagraph can reduce diffracted light imaged with a Keck-like pupil with $6 \mathrm{~mm}$ inter-segment gaps to less that $10^{-7}$ of its peak unocculted value (after accounting for Lyot stop losses), in the absence of phase aberrations. This is the expected level of residual uncorrected light from a spatially-filtered wavefront sensing adaptive optics system operating at 4-5 kHz, with 64 sensing channels across the Keck pupil, feeding an $H$-band science instrument (Poyneer and Macintosh ${ }^{6}$ ). The power in the coronagraphic PSF varies roughly as the square of the inter-segment gap width in the regimes we studied. The inner working distance of the coronagraph is of the order of $6-8$ diffraction-widths. 
Further refinement of the coronagraph design, or alternative designs such as the apodized pupil Lyot coronagraph (APLC, (Aime et al., ${ }^{11}$ Soummer et al. ${ }^{12}$ )) still need to be studied before we select a design. Apodized pupils have the advantage of effecting considerable speckle suppression, especially of first order and other pinned speckles (Bloemhof et al., ${ }^{17}$ Sivaramakrishnan et al. ${ }^{18}$ ) which may result in better PSF suppression (Perrin et al. ${ }^{19}$ ) and reduced speckle noise (Aime and Soummer ${ }^{20}$ ) than band-limited designs can provide close to the bright central source. It is also easier to see how we might control non-common path error between the coronagraph and the AO system with the APLC design, using light from inside the occulter. However, BLC's are easy to understand from a mathematical point of view, so we chose the BLC as the first design to explore for a Keck eXtreme Adaptive Optics Planetary Imager.

\section{ACKNOWLEDGMENTS}

R.S. and M.D.P. are supported by NASA Michelson Postdoctoral and Graduate Fellowships, respectively, under contract to the Jet Propulsion Laboratory (JPL) funded by NASA. The JPL is managed for NASA by the California Institute of Technology, and J.P.L. is supported by the California Institute of Technology's Millikan fellowship. We acknowledge support from the Space Telescope Science Institute's Director's Discretionary Research Fund, Research Programs Office, and Visitors Program, NSF grant AST-0088316, the National Science Foundation Science and Technology Center for Adaptive Optics, managed by the University of California at Santa Cruz under cooperative agreement No. AST-9876783.

\section{REFERENCES}

1. J. P. Lloyd, "The detection and characterization of low mass companions to sun-like stars," Ph.D. Thesis, 2002.

2. J. E. Nelson, "Design concepts for the California Extremely Large Telescope (CELT)," in Proc. SPIE Vol. 4004, p. 282-289, Telescope Structures, Enclosures, Controls, Assembly/Integration/Validation, and Commissioning, Thomas A. Sebring; Torben Andersen; Eds., pp. 282-289, Aug. 2000.

3. P. Dierickx and R. Gilmozzi, "Progress of the OWL 100-m telescope conceptual design," in Proc. SPIE Vol. 4004, p. 290-299, Telescope Structures, Enclosures, Controls, Assembly/Integration/Validation, and Commissioning, Thomas A. Sebring; Torben Andersen; Eds., pp. 290-299, Aug. 2000.

4. G. Chanan and M. Troy, "Strehl Ratio and Modulation Transfer Function for Segmented Mirror Telescopes as Functions of Segment Phase Error," Applied Optics 38, pp. 6642-6647, Nov. 1999.

5. N. Yaitskova and K. Dohlen, "Tip-tilt error for extremely large segmented telescopes: detailed theoretical point-spread-function analysis and numerical simulation results," Optical Society of America Journal 19, pp. 1274-1285, July 2002.

6. L. A. Poyneer and B. A. Macintosh, "Spatially filtered wave-front sensor for high-order adaptive optics," Journal of the Optical Society of America A 21, pp. 810-819, May 2004.

7. B. Lyot, "La couronne solaire etudie en dehors des eclipses," C. R. Acad. Sci Paris 191, p. 834, 1930.

8. B. Lyot, "The study of the solar corona and prominences without eclipses (George Darwin Lecture, 1939)," MNRAS 99, p. 580, June 1939.

9. A. Sivaramakrishnan, C. D. Koresko, R. B. Makidon, T. Berkefeld, and M. J. Kuchner, "Ground-based Coronagraphy with High-order Adaptive Optics," Astrophysical Journal 552, pp. 397-408, May 2001.

10. C. Aime, R. Soummer, and A. Ferrari, "Interferometric apodization of rectangular apertures. Application to stellar coronagraphy," Astronomy \&3 Astrophysics 379, pp. 697-707, Nov. 2001.

11. C. Aime, R. Soummer, and A. Ferrari, "Total coronagraphic extinction of rectangular apertures using linear prolate apodizations," Astronomy \& Astrophysics 389, pp. 334-344, July 2002.

12. R. Soummer, C. Aime, and P. Falloon, "Stellar coronagraphy with prolate apodized circular apertures," Astronomy \& Astrophysics 397, pp. 1161-1172, 2003.

13. J. W. Goodman, Introduction to Fourier Optics, McGraw Hill, New York, 1968.

14. B. A. Smith and R. J. Terrile, "A circumstellar disk around Beta Pictoris," Science 226, pp. 1421-1424, Dec. 1984. 
15. M. J. Kuchner and W. A. Traub, "A Coronagraph with a Band-limited Mask for Finding Terrestrial Planets," Astrophysical Journal 570, pp. 900-908, May 2002.

16. M. Troy and G. A. Chanan, "Diffraction effects from giant segmented-mirror telescopes," Applied Optics 42, pp. 3745-3753, July 2003.

17. E. E. Bloemhof, R. G. Dekany, M. Troy, and B. R. Oppenheimer, "Behavior of Remnant Speckles in an Adaptively Corrected Imaging System," Astrophysical Journal Letters 558, pp. L71-L74, Sept. 2001.

18. A. Sivaramakrishnan, J. P. Lloyd, P. E. Hodge, and B. A. Macintosh, "Speckle Decorrelation and Dynamic Range in Speckle Noise-Limited Imaging," Astrophysical Journal Letters 581, pp. L59-L62, Dec. 2002.

19. M. D. Perrin, A. Sivaramakrishnan, R. B. Makidon, B. R. Oppenheimer, and J. R. Graham, "The structure of high strehl ratio point-spread functions," Astrophysical Journal 596, pp. 702-712, Oct. 2003.

20. C. Aime and R. Soummer, "Usefulness and limits of coronagraphy in the presence of pinned speckles," Astrophysical Journal Letters (submitted), 2004. 